\title{
Nonlinear effects on the receptivity of crossflow in the swept Hiemenz flow
}

\begin{tabular}{|r|l|}
\hline Journal: & Journal of Fluid Mechanics \\
\hline Manuscript ID: & JFM-14-S-0439.R2 \\
\hline mss type: & Standard \\
\hline Date Submitted by the Author: & 24-Nov-2014 \\
\hline Complete List of Authors: & $\begin{array}{l}\text { Thomas, Christian; Imperial College London, Department of Mathematics } \\
\text { Hall, P.; Imperial College London, Mathematics } \\
\text { Davies, Christopher; Cardiff University, Mathematics }\end{array}$ \\
\hline Keyword: & $\begin{array}{l}\text { Boundary layer receptivity < Boundary Layers, Nonlinear instability < } \\
\text { Instability, Boundary layer stability < Boundary Layers }\end{array}$ \\
\hline & \multicolumn{2}{|l}{} \\
\hline
\end{tabular}

SCHOLARONE ${ }^{\text {IM }}$

Manuscripts 


\title{
Nonlinear effects on the receptivity of crossflow in the swept Hiemenz flow
}

\author{
By CHRISTIAN THOMAS ${ }^{1}$, PHILIP HALL ${ }^{1}$ and CHRISTOPHER \\ DAVIES $^{2}$ \\ ${ }^{1}$ Department of Mathematics, South Kensington Campus, Imperial College London, London, \\ SW7 2AZ, UK ${ }^{2}$ School of Mathematics, Cardiff University, Cardiff, CF24 4AG, UK
}

(Received ?? and in revised form ??)

Nonlinear effects on the receptivity of crossflow in the swept Hiemenz boundary layer are investigated. Numerical simulations are generated using a vorticity form of the NavierStokes equations. Steady perturbations are established using surface suction and blowing distributed along the spanwise direction as either a periodic strip or a band of small holes. The method of excitation, size and the location of the prescribed forcing are shown to have a significant influence on the receptivity of the boundary layer. Blowing holes are found to excite perturbations with considerably larger magnitudes than those generated using a periodic suction/blowing strip. A semi-log law relationship is derived that relates the initial amplitude of the linear only disturbances with the location that the absolute magnitude of the chordwise primary Fourier harmonic attains a stationary point or a size of approximately one tenth of the freestream spanwise velocity. Furthermore, the size of the physical chordwise velocity perturbation about this position can be estimated directly from the linear only solutions. This would suggest that for sufficiently small initial amplitudes the onset of some nonlinear flow development properties can be predicted directly from a linear receptivity analysis.

\section{Introduction}

Crossflow instability is a primary mechanism for the breakdown of the laminar flow and onset of transition in three-dimensional swept wing boundary layers. The disturbance is inflectional and is therefore susceptible to the inviscid instabilities examined by Gregory, Stuart \& Walker (1955). Taking the form of stationary co-rotating vortices (and traveling disturbances) crossflow has been observed on rotating disks and cones by Malik (1986) and Kohama (1984) amongst many others.

Many experimental and theoretical investigations have been undertaken concerning the various stages of the crossflow vortex development on swept wings. Using a swept wing model with an imposed pressure gradient Müller \& Bippes (1988) observed both stationary crossflow vortices and traveling wave disturbances prior to the onset of nonlinear saturation. Kohama, Saric \& Hoos (1991) identified a high-frequency secondary instability before the onset of transition. The linear and nonlinear theoretical investigations of three-dimensional boundary-layers were carried out by Meyer \& Kleiser (1988) using direct numerical simulations of the Navier-Stokes equations, while Fischer \& Dallmann (1991) applied a secondary instability analysis. Malik, Li \& Chang (1994) conducted an investigation of the linear, nonlinear and secondary instability stages of the crossflow evolution and the laminar-turbulent transition mechanisms in the swept Hiemenz flow. Their analysis was based on a system of nonlinear parabolized stability equations (NPSE). Both 
the stationary and traveling crossflow disturbances were examined. Detailed descriptions of the vortex development, inflectional velocity profiles and the generation of the socalled half-mushroom structures were discussed. A high-frequency secondary instability was observed prior to the onset of transition, agreeing with the earlier experiments on a swept cylinder by Poll (1985) and swept wing by Kohama et al. (1991). Similarly, in the rotating-disk boundary-layer, theoretical and experimental observations of a highfrequency secondary instability have been identified by the respective investigators Balachandar, Streett \& Malik (1992) and Kohama (1984). Further investigations concerning the development and control of secondary instabilities have been undertaken by Koch (2000), Janke \& Balakumar (2000), Wassermann \& Kloker (2003), Bonfigli \& Kloker (2007), Friederich \& Kloker (2012) and Obrist, Henniger \& Kleiser (2012).

Transition prediction in swept wing configurations are generally based on the $e^{N}$ methods that relate the onset of transition with the logarithmic amplitude ratio $N$ of the most unstable linear disturbance. Although this approach has many benefits and is implemented by both the academic and industrial communities it is only based on linear stability analysis and does not take into account the nonlinear effects, secondary instability or the initial amplitude of the disturbance within the boundary layer. Experimental measurements by Reibert, Saric, Carillo \& Chapman (1996) demonstrate that in order to accurately predict transition it is both necessary to consider the initial flow properties of the boundary layer disturbance and include the nonlinear development of the primary instability. Using NPSE methods, Malik, Li, Choudhari \& Chang (1999) generated a velocity profile for secondary instability analysis and found that the development of the stationary vortex agreed with the earlier experimental studies. The growth and frequency of the secondary instability prior to transition was consistent with the respective findings of Kohama, Onodera \& Egami (1996) and Kohama et al. (1991). Malik et al. (1999) describe an $N$ factor transition prediction method based on the secondary instability analysis that may be more efficient than a scheme that is reliant on the linear analysis

Environmental factors can filter into the boundary layer near a solid surface, seeding steady and unsteady fluctuations of the basic state. This process is known as boundary layer receptivity (Morkovin 1969) and characterises the initial stages of the breakdown of the laminar flow. External causes for receptivity can be attributed to the flow interacting with freestream acoustics, turbulence, vortices and wall deformations, including surface curvature, discontinuities and roughness (Saric, Reed \& Kerschen 2002). Receptivity of the boundary layer establishes the initial conditions for the amplitude, frequency and the phase of the disturbance within the flow.

If the initial amplitude of the perturbation remains relatively weak, the path to transition is driven by the excitation and development of a primary modal instability; TS waves on a flat plate geometry and crossflow on swept surfaces. Modal growth of the initially small amplitude disturbance can be computed using linear stability theory, but as the magnitude of the TS wave or crossflow instability grows, nonlinear processes are amplified through modal interaction with the higher order harmonics. This in turn can lead to the generation of secondary instabilities and transition (Herbert 1988; Saric, Reed \& White 2003).

For the current investigation our interest concerns the receptivity of crossflow disturbances within the swept Hiemenz boundary layer that develop through both the linear and nonlinear stages of the laminar-turbulent transition process. Crossflow disturbances are excited using two wall forcing schemes; a periodic suction/blowing strip and a band of periodic holes with constant suction or blowing. The form of crossflow generated by the wall forcing is then characterized by several initial flow properties, including the Reynolds number, spanwise periodicity and time-frequency. Further, the shape, location and the 
size of the forcing influences the amplitude of the disturbance and can significantly affect the onset of transition. When the initial amplitude of the disturbance is relatively weak, linear stability theory is implemented to compute receptivity. Given this assumption it is sufficient to decompose perturbations in the form

$$
q(\mathbf{x}, t)=A_{c} \tilde{q}(\mathbf{x}, t)
$$

where $\tilde{q}$ specifies the linear growth and the spatial-temporal evolution of the instability, while $A_{c}$ is a constant defined as the initial or receptivity amplitude of the disturbance. When wall roughness is responsible for the excitation of perturbations, the size of $A_{c}$ has been shown by Streett (1998) and Mughal (2012) to vary significantly with the shape, height and localized distribution of the surface imperfection. If the initial size of the perturbation is relatively small, the linear stages of the disturbance development can continue far downstream of the prescribed wall forcing. However, as the magnitude of the initial forcing and amplitude $A_{c}$ increases, the appearance of nonlinear effects and transition are promoted.

Theoretical investigations concerning the receptivity of three-dimensional boundary layers to wall roughness have been undertaken by Federov (1988), Choudhari (1993), Choudhari (1994), Crouch (1993) and Ng \& Crouch (1999). Receptivity to wall roughness on a curved aerofoil in a compressible flow were investigated by Collis \& Lele (1999) by solving the linearized Navier-Stokes equations. Wassermann \& Kloker (2002) developed a vorticity formulation to examine the effects of nonlinearity in the incompressible flow over a swept flat plate. Studies by Schrader, Brandt \& Henningson (2009) and Schrader, Amin \& Brandt (2010) considered the receptivity of the flow over a swept flat plate to free stream turbulence and wall roughness. Spalart (1990) generated crossflow vortex structures using disturbance inputs at the attachment-line that characterized random noise, waves and wavepackets. Further studies concerning receptivity have been undertaken by Hunt \& Saric (2011), Tempelmann, Hanifi \& Henningson (2012a), Tempelmann, Schrader, Hanifi, Brandt \& Henningson (2012b), Hosseini, Tempelmann, Hanifi \& Henningson (2013) and Lovig, Downs \& White (2014).

A vorticity formulation developed by Davies \& Carpenter (2001) is utilized and is extended to include the effects of nonlinearity on crossflow receptivity in the swept Hiemenz flow. Using the results of both the linear and nonlinear receptivity studies, relationships are sought that characterize the following flow properties:

i. The effect of nonlinearity on the size of the disturbance. Both locally about the centre of forcing and during the modal development of the crossflow instability.

ii. The onset of nonlinear saturation, where the linear and nonlinear disturbance computations are significantly different.

iii. The location where the primary Fourier harmonic of the chordwise velocity disturbance first attains a stationary point or maximum.

iv. The size of the physical disturbance about this location.

Details of the nonlinear extensions to the vorticity system of equations are included in the following section along with a description of the basic state. The numerical methods and computational parameter settings used to accurately and successfully generate crossflow are defined in $\S 3$. Definitions for the amplitude of the crossflow disturbance, the onset of nonlinear saturation and the chord location that the primary Fourier harmonic of the chordwise velocity attains a stationary point or maximum are given in $\S 4$. In $\S 5$ the receptivity of crossflow to various initial forcing distributions are discussed and final remarks are given in $\S 6$. 


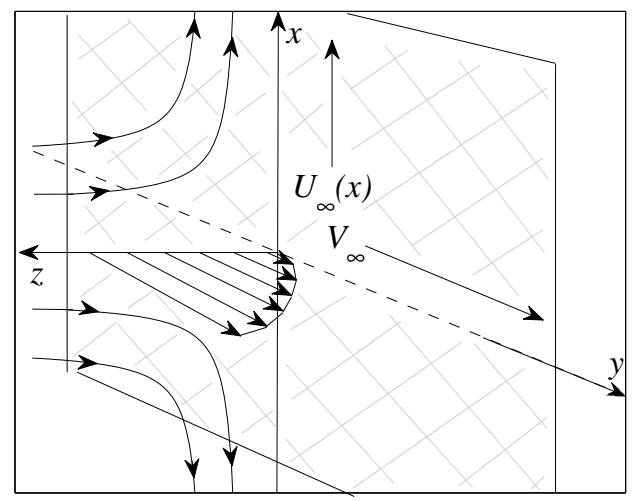

FIGURE 1. Illustration of the swept Hiemenz flow, depicting the streamlines in the chordwise $x$-direction and sweep velocity long the spanwise $y$-direction.

\section{Formulation}

\subsection{Swept Hiemenz Flow}

Consider an incompressible viscous fluid in a Cartesian coordinate system $\mathbf{x}^{*}=\left\{x^{*}, y^{*}, z^{*}\right\}$ relative to a swept plate, where $x^{*}$ and $y^{*}$ respectively denote the chordwise and spanwise flow directions. The $z^{*}$-axis specifies the direction normal to the wall. Uniform flow fields $U_{\infty}(x)=m x^{*}$ and $V_{\infty}$ are directed along the chordwise and spanwise axis, engineering the swept Hiemenz flow that impinges on an inclined plate. (Refer to figure 1 for an illustration of the described flow). The flow is symmetric about the attachment-line $x^{*}=0$ and for given conditions is susceptible to the disturbances investigated by Hall, Malik \& Poll (1984) and Spalart (1988). Further along the chord the flow is unstable to crossflow vortices that have been studied for their linear and nonlinear stability characteristics by Malik et al. (1994) amongst others.

Units of length are scaled on the typical thickness parameter $\delta=\sqrt{\nu / m}$ for $\nu$ the kinematic viscosity of the fluid. The corresponding velocity components are dimensionalized using the spanwise flow field $V_{\infty}$. Hence, the non-dimensional coordinate system and the undisturbed velocity are defined as

$$
\mathbf{x}=\mathbf{x}^{*} / \delta \text { and } \quad \mathbf{U}_{\mathbf{B}}=\mathbf{U}^{*} / V_{\infty} .
$$

Given the stream function

$$
\phi=x^{*} \sqrt{m \nu} f(z)
$$

and the boundary conditions

$$
\begin{aligned}
& \mathbf{U}^{*}=0 \quad \text { on } \quad z^{*}=0 \quad \text { and } \\
& U^{*} \rightarrow U_{\infty}, \quad V^{*} \rightarrow V_{\infty} \quad \text { as } \quad z^{*} \rightarrow \infty,
\end{aligned}
$$

solutions to the Navier-Stokes equations are given by the undisturbed velocity profiles

$$
U_{B}=\frac{x}{R} f^{\prime}(z), \quad V_{B}=g(z), \quad W_{B}=-\frac{1}{R} f(z) .
$$

The wall-normal functions $f$ and $g$ are computed by solving the system of ordinary differential equations

$$
f^{\prime \prime \prime}+f f^{\prime \prime}+\left(1-f^{\prime 2}\right)=0, \quad g^{\prime \prime}+f g^{\prime}=0,
$$


where prime denotes differentiation with respect to $z$. The Reynolds number of the flow system is defined as

$$
R=\frac{V_{\infty} \delta}{\nu}
$$

where the streamline angle $\theta$ is given by the expression

$$
\tan \theta=\frac{V_{\infty}}{U_{\infty}(x)} \equiv \frac{R}{x} .
$$

\subsection{Governing Disturbance Equations}

The nonlinear disturbance development and receptivity of the crossflow instability are investigated using a vorticity form of the Navier-Stokes equations. Total velocity and vorticity fields are decomposed as the sum of their undisturbed state (2.1) and perturbed parts:

$$
\mathbf{U}=\mathbf{U}_{\mathbf{B}}+\mathbf{u}, \quad \text { and } \quad \boldsymbol{\Omega}=\boldsymbol{\Omega}_{\mathrm{B}}+\boldsymbol{\omega},
$$

where the perturbation fields are denoted as

$$
\mathbf{u}=\{u, v, w\}, \quad \text { and } \quad \boldsymbol{\omega}=\left\{\omega_{x}, \omega_{y}, \omega_{z}\right\} .
$$

The system of governing equations for the swept Hiemenz flow are composed of the vorticity transport equation and the Poisson relationship:

$$
\begin{gathered}
\frac{\partial \boldsymbol{\omega}}{\partial t}+\nabla \wedge \mathbf{N}=\frac{1}{R} \nabla^{2} \boldsymbol{\omega}, \\
\nabla^{2} \mathbf{u}+\nabla \wedge \boldsymbol{\omega}=0,
\end{gathered}
$$

for $\mathbf{N}=\boldsymbol{\Omega}_{\mathbf{B}} \wedge \mathbf{u}+\boldsymbol{\omega} \wedge \mathbf{U}_{\mathbf{B}}+\boldsymbol{\omega} \wedge \mathbf{u}$. Perturbations are then divided into two subsets: principle $\left\{\omega_{x}, \omega_{y}, w\right\}$ and secondary variables $\left\{u, v, \omega_{z}\right\}$. The three principle variables are given as solutions of the $x$ and $y$ forms of (2.4a) coupled with the $z$ component of $(2.4 b)$. The remaining secondary variables are defined explicitly in terms of the principle fields by rearranging the definitions for vorticity and the solenoidal condition:

$$
\boldsymbol{\omega}=\nabla \wedge \boldsymbol{u} \text { and } \nabla \cdot \boldsymbol{\omega}=0 .
$$

Disturbances are assumed to be periodic along the spanwise $y$-direction. Hence, perturbation fields are represented using a Fourier series as

$$
q(\mathbf{x}, t)=\sum_{k=-\infty}^{\infty} q_{k}(x, z, t) e^{i k \beta y}
$$

where $\beta$ is the spanwise wavenumber of the perturbation. Here $k$ corresponds to the $k t h$ harmonic of the spanwise Fourier series. To simplify the problem and ease computational time restraints, it is sufficient to truncate the order of the Fourier series to only a finite number of modes

$$
q(\mathbf{x}, t)=\sum_{k=-N_{y}}^{N_{y}-1} q_{k}(x, z, t) e^{i k \beta y},
$$

where $N_{y}$ is an integer representing half the number of modes in the series.

\subsection{Excitation of the Undisturbed Flow}

Steady perturbations are established by forcing the undisturbed flow using suction or blowing imposed at the plate surface:

$$
u=v=0 \quad \text { and } \quad w=w_{w}(x, y, t) \quad \text { on } \quad z=0 .
$$




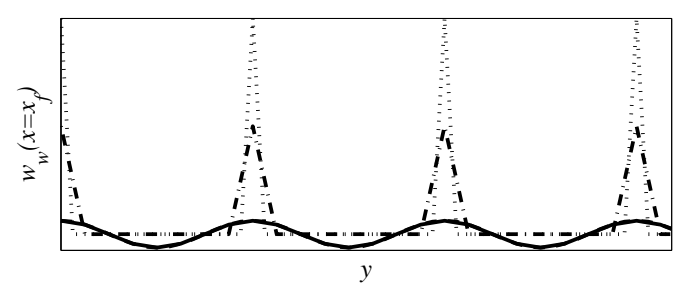

Figure 2. Physical spanwise shape of wall forcing at $x=x_{f}$. Method I represented as solid $\left(Y_{N}=4\right)$ and dashed lines (8), while chain (4) and dotted (8) correspond to method II with blowing $c$ positive.

The function $w_{w}$ is chosen to be both periodic along the spanwise direction and in time, so as to excite the desired stationary or traveling crossflow disturbance

$$
w_{w}=\sum_{k=-N_{y}}^{N_{y}-1} w_{w, k} h(x) e^{i(k \beta y-f t)},
$$

where $f$ is the temporal frequency, $w_{w, k}$ specifies the magnitude of the $k t h$ Fourier mode and the chord distribution $h=h(x)$ is defined as a normalized Gaussian function

$$
h(x)=\frac{1}{\sqrt{2 \pi}} e^{-\left(x-x_{f}\right)^{2} / 2} .
$$

Here $x_{f}$ is the chord centre of the wall forcing.

Suction and blowing are generated using two simple forcing models that are illustrated in figure 2 and characterized by the Fourier amplitudes:

$$
\begin{aligned}
\text { I. } \quad w_{w, 1}=c \quad \text { and } \quad w_{w, k}=0 \quad \forall \quad k \neq 1 \quad \text { and } \\
\text { II. } \quad w_{w, k}=c \quad \forall \quad k .
\end{aligned}
$$

Scheme I (solid and dashed lines respectively correspond to $N_{y}=4$ and 8 in figure 2) represents a periodic suction/blowing strip, where the sign of $c$ has no effect on the response of the crossflow disturbance. Only the primary mode is initially excited, but through the processes of nonlinear interaction the growth of the higher order harmonics are stimulated. All modes are forced equally by method II (chain and dotted lines denote cases $N_{y}=4$ and 8 in figure 2), which in the physical plane approximately represents a band of small point-like periodic holes prescribed with either wall suction (negative $c$ ) or blowing only (positive $c$ ). As the value of $N_{y} \rightarrow \infty$ the physical shape of the wall forcing will tend towards a Dirac-delta type function along the spanwise direction. Obviously for finite $N_{y}$ there will then be some inconsistencies between the disturbance amplitudes of solutions for different $N_{y}$ (which we will investigate further in subsequent sections). The size of the amplitude $c$ then imposes conditions on the receptivity analysis. For relatively small forcing amplitudes the disturbance will remain linear for the early stages of the flow development. However, as the magnitude of $c$ increases, the effects of nonlinearity will become significant and affect both the initial size of the disturbance and the downstream development of the flow system.

Although the two wall schemes are crude representations of wall suction/blowing and possibly physically unrealistic, they provide a simple means of testing the vorticity formulation and analysing the receptivity and development of crossflow disturbances to varying forms of forcing. Implementation of more complex and physically realistic suction holes can then be modeled (on Spalart 1990, 1993) by modifying the relative sizes of the Fourier harmonics $w_{w, k}$. 
The no-slip condition (2.8) is included in the vorticity formulation by deriving two integral constraints for the principle vorticity variables $\left\{\omega_{x}, \omega_{y}\right\}$. This is achieved by rearranging the secondary variable definitions $(2.5)$ for $u(\equiv 0)$ and $v(\equiv 0)$ as

$$
\begin{gathered}
\int_{0}^{\infty} \omega_{x} d z=\int_{0}^{\infty} \frac{\partial w}{\partial x} d z \text { and } \\
\int_{0}^{\infty} \omega_{y} d z=\int_{0}^{\infty} \frac{\partial w}{\partial y} d z .
\end{gathered}
$$

These two integral constraints replace the no-slip conditions $(2.8 a, b)$ along the $x$ and $y$ directions. When coupled with the wall-normal $z$ condition $(2.8 c)$, they provide the necessary set of boundary wall constraints on the above system of governing equations. Null conditions are imposed on perturbations at the upstream boundary, whilst at the opposite end of the spatial domain a wave-like condition is implemented that allows disturbances to propagate through the computational box without causing spurious reflection effects. Boundary conditions in the far-field limit are imposed on disturbance fields by ensuring that the three principle perturbation fields $\left\{\omega_{x}, \omega_{y}, w\right\}$ tend to zero as $z \rightarrow \infty$. (Further details are included within Davies \& Carpenter 2001).

\section{Numerical Methods}

\subsection{Discretization}

The numerical methods adopted for the discretization of the vorticity formulation are described as follows. Chordwise derivatives are represented using a fourth-order centred, compact finite difference approximation, while a Fourier expansion (outlined above in $\S 2.2)$ is implemented along the spanwise direction. Along the normal to wall axis a change in variable is implemented

$$
\xi=\frac{L}{L+z},
$$

to map points from the semi-infinite physical plane $z \in[0, \infty)$ onto a finite computational box $\xi \in(0,1]$. The mapping parameter $L=4$ prescribes the distribution of points along the wall-normal direction and was carefully chosen to ensure the resolution of the disturbance structures (crossflow and possible secondary instabilities) within and about the edge of the boundary-layer are accurately resolved. Disturbances are then expanded in terms of Chebyshev polynomials

$$
q_{k}(x, z, t)=\sum_{j=1}^{N_{z}} q_{k, j}(x, t) T_{j}(\xi),
$$

where $T_{j}$ is the $j$ th Chebyshev polynomial. Here $N_{z}$ is the order of the series truncation and $j$ is an odd and even integer for the respective principle and secondary variables. (Subscript $k$ refers to the value of the harmonic in the Fourier series). The system of governing equations (2.4) are then integrated twice with respect to the mapped variable $\xi$, generating a simple set of banded matrix representations for the wall-normal variation. Finally, the time-marching is treated using a combination of a predictor-corrector scheme and semi-implicit methods.

\subsection{Computational Parameter Settings}

Steady periodic crossflow instability was generated by carefully selecting the following discretization parameters: 
i. Number of wall-normal Chebyshevs $N_{z}$;

ii. Time step $\Delta t$;

iii. Chord grid step $\Delta x$;

iv. Chord domain size $N_{x}$;

v. Number of spanwise Fourier modes $N_{y}$.

As our investigation concerns the initial response of disturbances to periodic forcing, the above parameters were not constrained by the need to fully capture small-scale structures that could evolve following the development of nonlinear flow characteristics. Thus, well resolved and numerically stable results (up to the chord location that small-scale effects first appear) were successfully obtained by prescribing step sizes $\Delta t=0.01$ and $\Delta x=0.5$. Further it was deemed sufficient to set the number of Chebyshev polynomials $N_{z}=48$. Figure 3(a) illustrates the effectiveness of this parameter selection by comparing contours of the chordwise primary Fourier harmonic $u_{1}$ in the $(x, z)$-plane, which were established using the forcing method $\mathbf{I}$. The suction/blowing forcing was prescribed a zero frequency $(\omega=0)$, so that stationary crossflow instabilities could develop, while the spanwise wavenumber $\beta=0.4$ and the Reynolds number $R=500$. The Gaussian distribution along the chord direction was centred about the position $x_{f}=186$ with an amplitude $c=0.01$. The disturbance evolution is depicted over a short chordwise region, upstream of the onset of full nonlinear flow development. Solid lines display contours of the solution generated using the above parameter settings, while the dashed and dotted lines respectively denote solutions for the settings $\left\{\Delta x, N_{z}\right\}=\{0.25,48\}$ and $\{0.5,64\}$. All three sets of contour lines are indistinguishable up to the chordwise location $x=400$, which as we will observe in the subsequent discussion on receptivity is downstream of the region of interest for this particular problem.

The length of the chordwise domain was varied inversely proportional with the initial amplitude $c$ of the suction and/or blowing forcing. This ensured that disturbances of varying initial magnitude had sufficient chord length to develop. For the largest values of $c(\sim 0.05)$ considered, the size of $N_{x}$ was limited to approximately $400 \delta$ chord units beyond the forcing location $x_{f}$, which was increased to $800 \delta$ chord units for the smallest values of $c$.

The final parameter necessary to generate successful and reliable nonlinear simulation results was the number of spanwise Fourier modes $N_{y}$. Figure 3 compares the maximum amplitudes of the Fourier $u_{k}$-velocity perturbations for $N_{y}=4$ (solid lines) and 8 (dashed) modes. Linear only results are included and plotted using dotted lines for purposes of comparing with the solutions of the nonlinear investigation. The flow specifications are as given above $\{\omega, \beta, R\}=\{0,0.4,500\}$ for $\left\{x_{f}, c\right\}=\{186,0.01\}$. Steady disturbance amplitudes generated by method $\mathbf{I}$ are depicted in figure $3(\mathrm{~b})$, while figure 3 (c) illustrates results driven by forcing scheme II. Disturbances excited by $\mathbf{I}$ for $N_{z}=4$ and 8 exhibit very minor variations that are barely noticeable on the given plot resolution. The two primary Fourier velocity components, $\left|u_{1}\right|_{\max }$, are identical over the chord length considered and deviations from the linear result only emerge for $x>300$.

Results generated using the second forcing method do not display such consistent behaviour as there are relatively large differences between the two nonlinear and linear only simulation results that appear almost immediately about the origin of forcing. There are also small discrepancies between the magnitudes of the two nonlinear calculations. Although these differences are marginal, they may be augmented as the amplitude $c$ of the initial forcing is increased to larger values. This behaviour is to be expected for method II as it is clearly illustrated in figure 2 that the physical representation of the point like forcing can vary quite significantly for variable $N_{y}$. Further nonlinear simulations were generated for larger $N_{y}$ Fourier modes, with results (discussed in subsequent sec- 
(a)
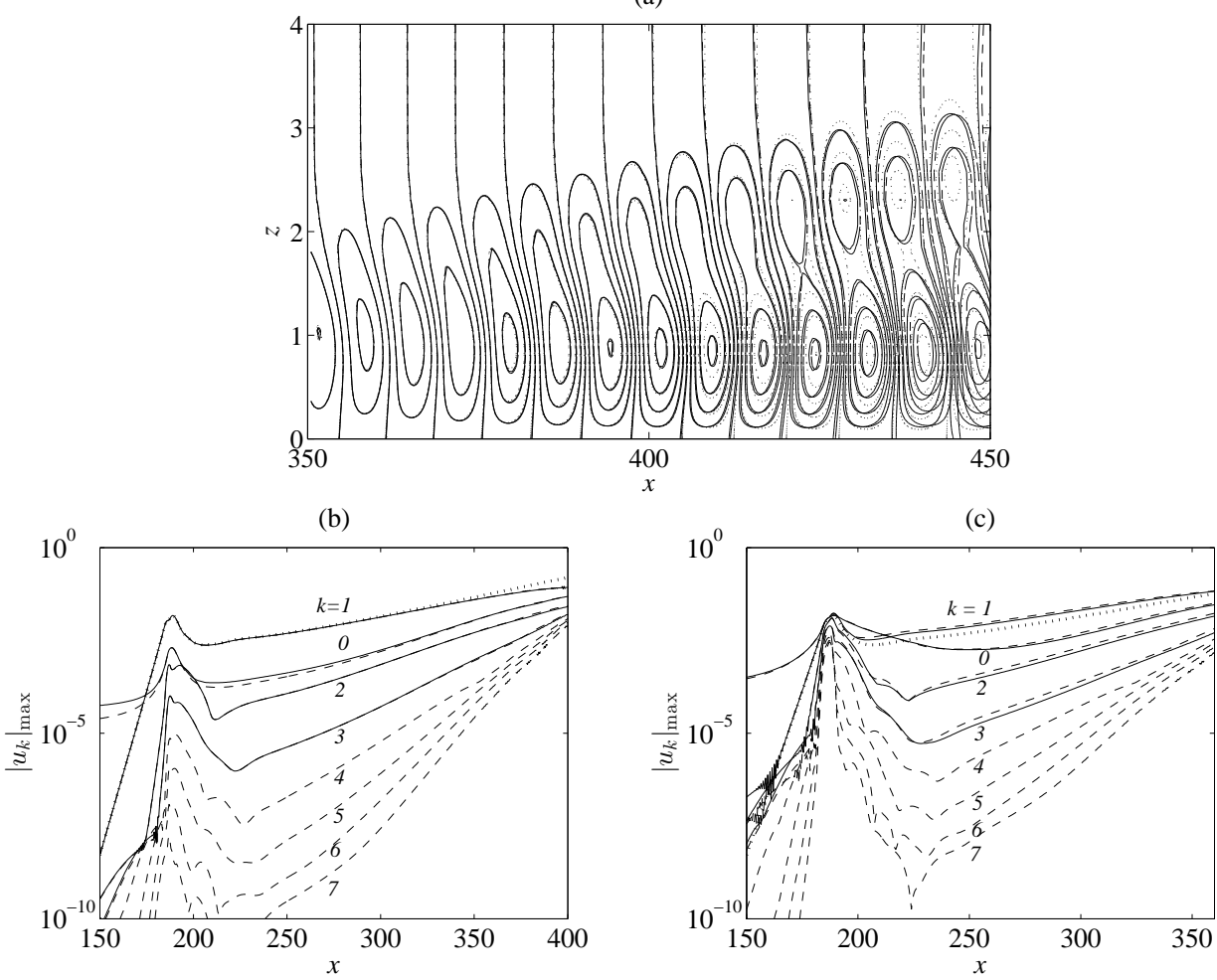

Figure 3. (a): Contours of the primary $u_{1}$-velocity Fourier harmonic for $\left\{\Delta x, N_{z}\right\}=\{0.5,48\}$ (solid) $\{0.25,48\}$ (dashed) and $\{0.5,64\}$ (dotted). (b, c): Maximum amplitudes of the $N_{y}$ Fourier $u_{k}$-velocity fields for forcing methods $\mathbf{I}(\mathrm{a})$ and $\mathbf{I I}(\mathrm{b})$ and amplitude of forcing $c=0.01$. The Reynolds number $R=500$, spanwise wavenumber $\beta=0.4$ and centre of forcing $x_{f}=186$. Solid lines indicate results generated using $N_{y}=4$ and dashed correspond to $N_{y}=8$ modes. (Dotted lines illustrate the linear only results.)

tions regarding receptivity and nonlinear flow development) suggesting that additional harmonics has a relatively small effect on the size and evolution of the disturbance.

For the greater part of this investigation it was decided that $N_{y}=4$ Fourier modes would be sufficient to study the general effects of nonlinearity on the receptivity and development of crossflow. The primary reason behind the choice of $N_{y}$ was to save on both computational resources and time expenditure. Further it was suggested by Malik et al. (1994) that the nonlinear characteristics of the crossflow vortex could be modeled successfully using only the primary Fourier mode and its zeroth and second order harmonics. Although computations generated by method II would inevitably vary for additional Fourier harmonics, (as the physical representations of the wall forcing are different for variable $N_{y}$ ), differences in disturbance amplitude and flow characteristics were relatively minimal, even for relatively large initial forcing sizes $c$. Further discussion of the effect of increasing $N_{y}$ is included below with results documented in tables 1 and 2 . 


\section{Disturbance Definitions}

In parabolized stability equation analysis, linear crossflow disturbances are assumed to take the form

$$
q(\mathbf{x}, t)=A_{c} \tilde{q}(x, z) e^{\int_{x_{0}}^{x} \alpha\left(x^{\prime}\right) d x^{\prime}+i\{\beta y-\omega t\}},
$$

where $\alpha$ is the chord wavenumber of the perturbation and $x_{0}$ corresponds to the location for neutral stability. Here $\tilde{q}$ is a shape function that varies slowly along the chord direction and is normalized about $x_{0}$. The magnitude of the disturbance is represented by the real constant $A_{c}$, which is described herein as the receptivity amplitude, for subscript $c$ the size of the initial forcing. For a linear only analysis the magnitude of $A_{c}$ is directly proportional to the size of $c$ as

$$
A_{c}=c A_{1}
$$

where $A_{1}$ is the amplitude of a crossflow disturbance associated with $c=1$. For the current investigation it is convenient to assume that linear disturbances are also of the form (4.1), as it provides an effective means of extracting the wavenumber, growth rate and disturbance amplitude from the vorticity formulation computations.

\subsection{Receptivity Amplitude}

By neglecting the slow chordwise variation of the shape function, setting $\tilde{q}(x, z) \approx \tilde{q}(z)$, the definition for linear crossflow can be simplified to the form

$$
q(\mathbf{x}, t)=A_{c} \tilde{q}(z) e^{\int_{x_{0}}^{x} \alpha\left(x^{\prime}\right) d x^{\prime}+i\{\beta y-\omega t\}},
$$

where we assume $|\tilde{q}|=1$. Further, introducing a normalisation factor, equation (2.3), and replacing the function $q$ with the primary Fourier chordwise perturbation field $u_{1}$, we set

$$
M(x)=\max _{z}\left|u_{1}(x, z)\right| \frac{R}{x} .
$$

A definition for the absolute value of the receptivity amplitude is then given as

$$
A_{c}=\frac{M(x) \frac{x}{R}}{\left|e^{\int_{x_{0}}^{x} \alpha(x) d x}\right|} .
$$

For a prescribed set of initial flow conditions, $\{\omega, \beta, R\}$, the exponential denominator is a fixed $x$-dependent function that is not related to the choice of wall forcing. Thus, once the field $u_{1}$ is generated the receptivity amplitude $A_{c}$ of the disturbance can be calculated. Though this expression is only applicable to linear crossflow disturbances, we will also apply it to solutions of the nonlinear investigation, as it provides a means of comparing the two sets of results.

\subsection{Saturation}

The location that the growth rate of the nonlinear disturbance first differs significantly from that given by the linear calculation is known as saturation; the interaction of the primary mode with the higher order harmonics has become sufficiently large to impact on the disturbance evolution and the growth of the system. In an attempt to quantify nonlinear saturation we define

$$
K_{*}(x)=\frac{M_{x}}{M},
$$

where the subscript $x$ refers to the chordwise derivative, while the subscript for $K$ is either $L$ for linear analysis or $N L$ for the corresponding nonlinear theory computations. 
The appearance of nonlinear saturation is then defined here as satisfying the expression

$$
F(x) \equiv\left|\frac{K_{N L}-K_{L}}{K_{L}}\right| \times 100 \%>1 \%,
$$

which was decided upon (following the analysis given in subsequent sections) as being a sufficiently large percentage difference to delineate between the linear and nonlinear results. Further, it was assumed that the linear disturbance function $K_{L}$ is non-zero and at the very least represents a convectively growing disturbance.

\subsection{Stationary Point}

Finally, we introduce a definition that characterises the chordwise location that the absolute value of $M$ first attains a stationary point or peak value:

$$
M_{x}=K_{N L}=0 \quad \text { and } \quad F(x)=100 \%,
$$

which coincides with $M$ typically having a magnitude of approximately one tenth of the spanwise freestream velocity $V_{\infty}$. Further, this location approximately coincides with the appearance of small-scale structures that arise with the emergence of nonlinear flow development.

\section{Results}

\subsection{Disturbance Development and Growth}

A steady stationary crossflow disturbance was generated for approximately the strongest growing spanwise wavenumber $\beta=0.4$, while the Reynolds number of the flow $R=$ 500 is chosen as it is smaller than the critical value for the attachment-line instability $(R \sim 583)$ and allows easier comparison with solutions of earlier investigations (Malik et al. 1994). Forcing scheme $\mathbf{I}$ was centred about the chordwise location $x_{f}=186$ with amplitude $c=0.01$. The periodic suction/blowing strip was then switched on at time $t=0$, triggering the excitation of disturbances within the boundary layer. Downstream of the forcing a crossflow instability evolves downstream along the chordwise direction.

Figure 4 depicts cross-sectional cuts of the total $U$-velocity in the $(y, z)$-plane, plotted using labeled line contours at four successive positions along the chordwise direction; $x=200,300,400$ and 450 . The flow dynamics are plotted over two spanwise wavelengths where the $z$-axis is stretched for visualisation purposes. Contours are plotted from a value of zero at the wall through to a maximum magnitude of approximately 0.9 in the far-field. Crossflow vortices are observed to evolve into the shapes described by Malik et al. (1994) as half-mushroom structures. About $x=200$ the boundary layer thickness remains relatively constant along the length of the spanwise direction, as there are only small variations in the total velocity. However, at larger chord positions, the nonlinear effects emerge and the disturbance rolls into vortices, creating regions of low and high velocity. This in turn results in significant variations in the boundary-layer thickness along the span. The evolution of the crossflow vortex is comparable with that described by Malik et al. (1994), even though only four spanwise Fourier modes are used herein to generate the disturbance.

Figure 5(a) displays the growth rates of several linear and nonlinear disturbances calculated using the formula

$$
\alpha=\frac{1}{u_{1}} \frac{\partial u_{1}}{\partial x}
$$


(a)

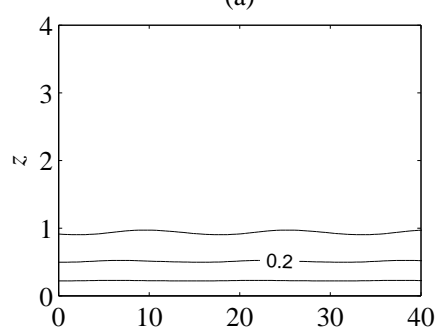

(c)

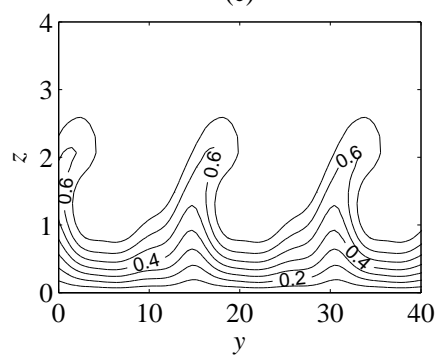

(b)

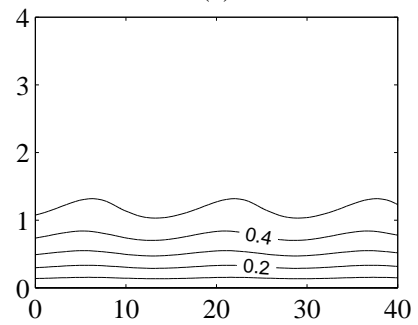

(d)

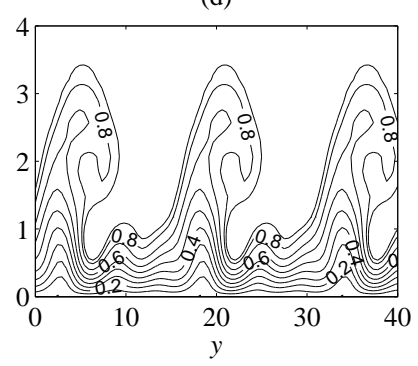

Figure 4 . Total chordwise velocity in the $(y, z)$-plane at $x=200,300,400$ and 450 , for $\beta=0.4$ and $R=500$.

where $u_{1}$ is the primary Fourier velocity component in the chordwise direction. The dotted line depicts the growth rate of the linear only simulation, while the solid, dashed and chain lines represent the growth rates of the nonlinear primary mode, where the initial forcing $c=0.03,0.01$ and 0.001 , respectively. In all four cases the periodic suction/blowing strip is centred about $x_{f}=186$. In the chord region immediately downstream of the wall forcing, a short transient stage is observed, which is attributed to several perturbations initially competing to influence the response of the flow. However, the stationary crossflow disturbance is eventually observed and the growth rates follow a set path. The linear growth rate behaves in a manner consistent with the observations of earlier studies on non-parallel linear effects; the growth rate attains a maximum value of approximately 0.25 near $x=350$ and slowly decreases thereafter. The development of the three nonlinear growth rates also follow a similar evolutionary path. However, as $c$ increases, the growth rate is found to saturate and deviate from the linear solution at varying chord locations. This is observed about the chord positions $x=250$ and 370 for the respective forcing amplitudes $c=0.01$ (dashed) and 0.001 (chain). These chord locations are also consistent with the calculations of the function $F$, equation $(4.5 b)$, displayed here in figure $5(\mathrm{~b})$. Line types match those given for the above nonlinear solutions, while the horizontal dotted line now represents the suggested $F=1 \%$ difference for the onset of nonlinear saturation. For the largest forcing amplitude, $c=0.03$, significant differences between the linear and nonlinear growth rates appear directly about the centre of excitation with a minimum value for $F \approx 3 \%$. This would suggest that this represents a rather large magnitude suction/blowing wall forcing. Thus, for greater initial forcing amplitudes the linear stages of the disturbance development may be bypassed altogether.

\subsection{Absolute Perturbation Velocity}

Figure 6 displays the maximum amplitude of the primary Fourier component of the $\bar{u}$ velocity against the chordwise $x$-direction, where equation (4.3) for $M$ has been utilised to 

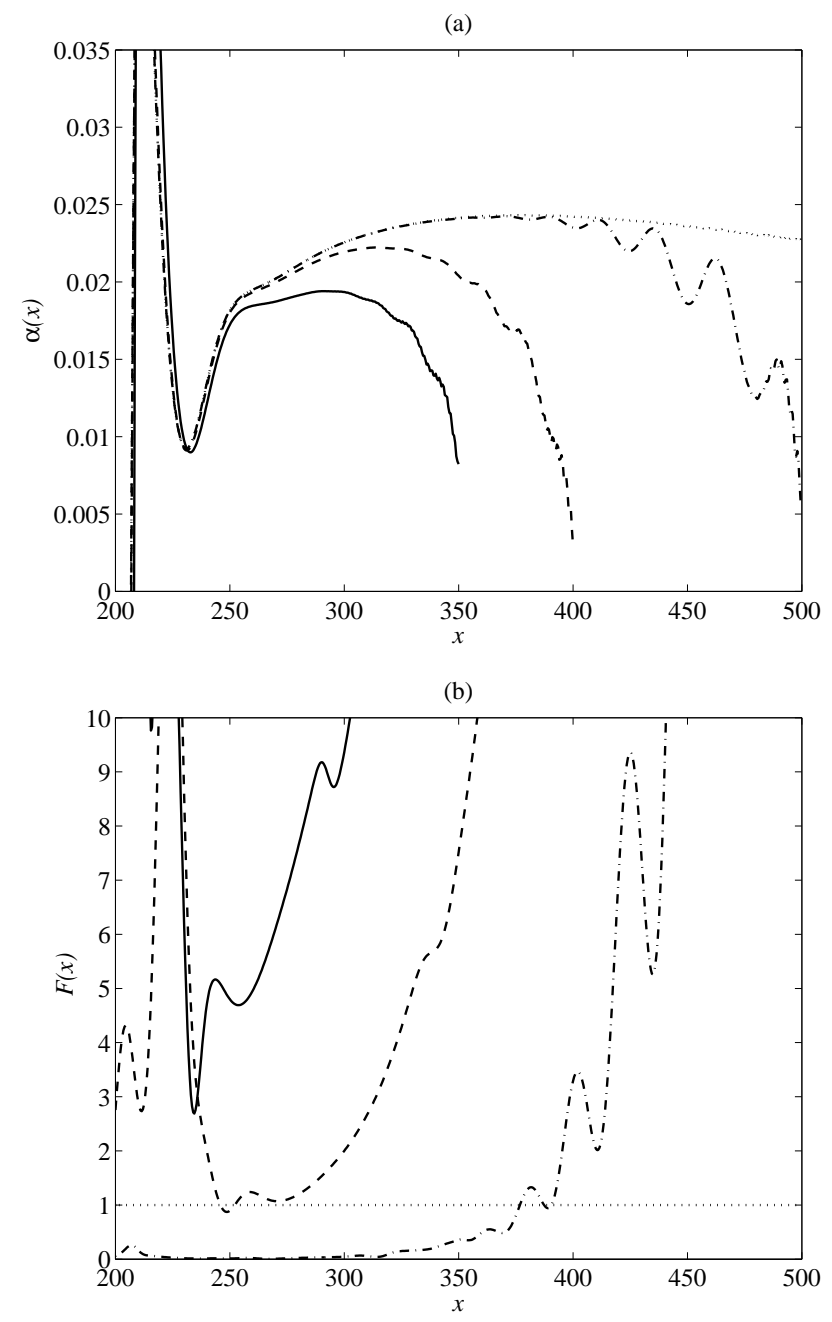

FiguRE 5. (a): Disturbance growth rate for $\beta=0.4$ and $R=500$. Nonlinear primary mode with $c=0.03$ (solid line), 0.01 (dashed) and 0.001 (chain). The dotted curve corresponds to a linear only disturbance. (b): Saturation prediction for the disturbances given in (a), with the formula based on that defined in $(4.5 b)$.

simplify notation and illustrate solutions. The first plot shows results over an extended chord range, from upstream of the periodic forcing through to the location that the disturbance first attains a stationary or peak point (as outlined in $\S 4.3$ ). The second illustration presents results centred about the location of the periodic wall forcing. A semi-log law scaling has been applied along the vertical axis to assist visualization and interpretation.

The solid line depicts the disturbance solution established by forcing method $\mathbf{I}$ for $c=0.01$, where only the primary disturbance is excited. However, through nonlinear the nonlinear processes the higher order harmonics are generated and forced to grow proportionally with the primary mode. The nonlinear solution exhibits very small variations to the response of the linear computation (drawn using a dotted line). Comparing the perturbation development over an elongated chordwise frame (figure 6(a)) would suggest 

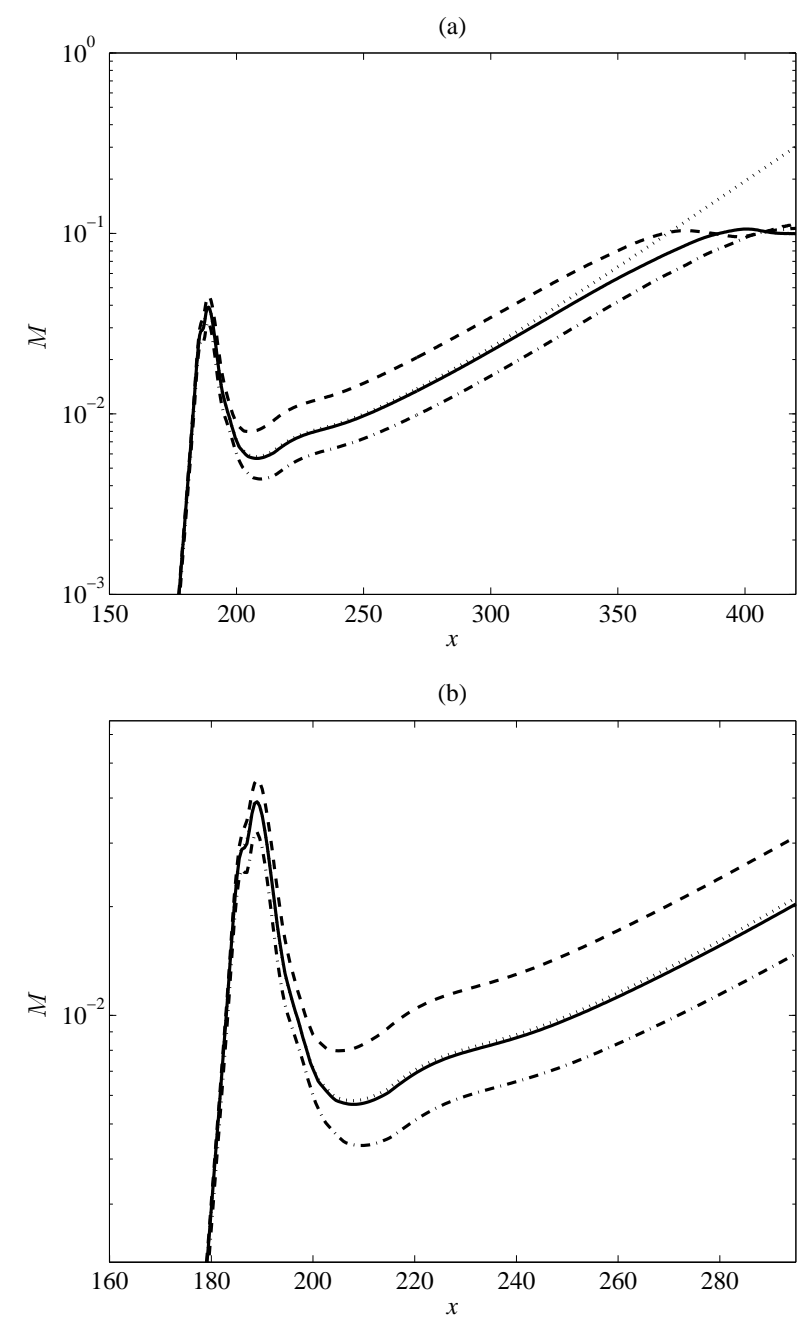

Figure 6. Maximum amplitudes $M$ for forcing schemes $\mathbf{I}_{c=0.01}$ (solid), $\mathbf{I I}_{c=0.01}$ (dashed) and $\mathbf{I I}_{c=-0.01}$ (chain). The Reynolds number of the flow $R=500$, the spanwise wavenumber $\beta=0.4$ and centre of forcing $x_{f}=186$. The dotted line represents the linear only solution. (a): Extended chord range. (b): Localized about the centre of forcing.

that significant variations in magnitude only appear for $x>300$. Indeed, the localized view of the perturbation in figure $6(\mathrm{~b})$ indicates that the amplitude change about the wall forcing is relatively insignificant. This suggests that, in this instance, the nonlinear effects have very little impact on the receptivity of the disturbance and only appear through the modal interaction of the downstream development of the crossflow vortex.

Dashed and chain lines depict simulation results driven by scheme II where $c=0.01$ (blowing only) and $c=-0.01$ (suction), respectively. In these particular cases all Fourier modes are now excited equally, but the higher order harmonics are quickly forced to grow relative to the primary perturbation. Amplitudes of the primary velocity components seeded using the wall forcing method II show a considerable variation from the linear only simulation; both locally about the origin of the forcing and the effective size of the disturbance that evolves downstream. Blowing increases the size of the disturbance, 


\begin{tabular}{ccc}
$N_{y}$ & $\max |M|_{x=x_{f}}$ & $E_{1} \%$ \\
\hline Linear & 0.039355 & 0.0 \\
4 & 0.044841 & 12.2 \\
6 & 0.044926 & 12.4 \\
8 & 0.045049 & 12.6 \\
10 & 0.045285 & 13.1 \\
12 & 0.045552 & 13.6
\end{tabular}

TABLE 1. Comparing maximum amplitudes of the primary Fourier harmonic $u_{1}$ at the origin of forcing $x_{f}$ for disturbances with mode numbers $N_{y}=4,6, \ldots, 12$. Disturbances are generated using forcing scheme II and $c=0.01$. The parameter $E_{1}$ specifies the relative differences between the linear and nonlinear solutions.

whilst suction shows a comparable reduction in the perturbation magnitude. Hence, the effects of nonlinearity are now very relevant to the receptivity process, as well as in the crossflow evolution. These observations agree with the original findings of Choudhari \& Duck (1996), who first noticed that the onset of nonlinearity could initially emerge in the receptivity stages of the flow development, very much before the subsequent appearance of nonlinear processes in the crossflow disturbance.

The effect of nonlinearity on the receptivity at the point of forcing $x_{f}$ is presented in table 1 . The relative amplitudes of the primary mode $u_{1}$ are compared for varying Fourier harmonics $N_{y}$, where results correspond to those disturbances generated using the blowing only forcing (method II) for $c=0.01$. Nonlinearity increases the relative size of the perturbation about $x_{f}$, but the percentage difference between the $N_{y}=4$ and 12 mode cases is very small, suggesting that our earlier assumption that four spanwise modes may be adequate for investigating crossflow receptivity. Nevertheless, nonlinearity has affected the local receptivity and size of the disturbance about the centre of wall forcing. However, in subsequent analysis we will observe that nonlinearity has an even greater impact on the effective receptivity amplitude of the developing crossflow instability.

The absolute maximums of the three disturbances attain a stationary point or peak magnitude about a value of 0.1 , near the chord locations $x=370,390,410$ for forcing schemes $\mathbf{I I},_{c=0.01}, \mathbf{I}_{c=0.01}$ and $\mathbf{I I}_{c=-0.01}$, respectively. Downstream of the stationary point the maximum value of the primary mode is found to fluctuate about this amplitude. Small scale structures are then observed to develop, but due to the prescribed discretization (discussed earlier) are not captured to any degree of certainty. However, as the current investigation only concerns the effects of nonlinearity on receptivity and the early stages of disturbance evolution, any resolution errors beyond this stationary point can be safely ignored.

Additional plots of maximum disturbance amplitudes $M$ are presented in figure 7 . The line types are the same as those given in figure 6 . The first illustration (figure $7(\mathrm{a})$ ) depicts the effect of varying the forcing amplitude $c$ on the receptivity of the disturbance. Two forcing amplitudes are considered: $c=0.03$ in the upper half of the plot and $c=0.001$ in the lower half. There are no discernible differences between the perturbation amplitudes generated using the smaller forcing (at least for $x<400$ ). However, those disturbances excited by the larger forcing amplitude are observed to display significant deviations from the linear only response. This also includes the crossflow disturbance established by wall forcing method $\mathbf{I}$, which now displays distinct variations in magnitude. Further, the size of $M$, for the suction/blowing strip and blowing only forcing, is greater about the centre 

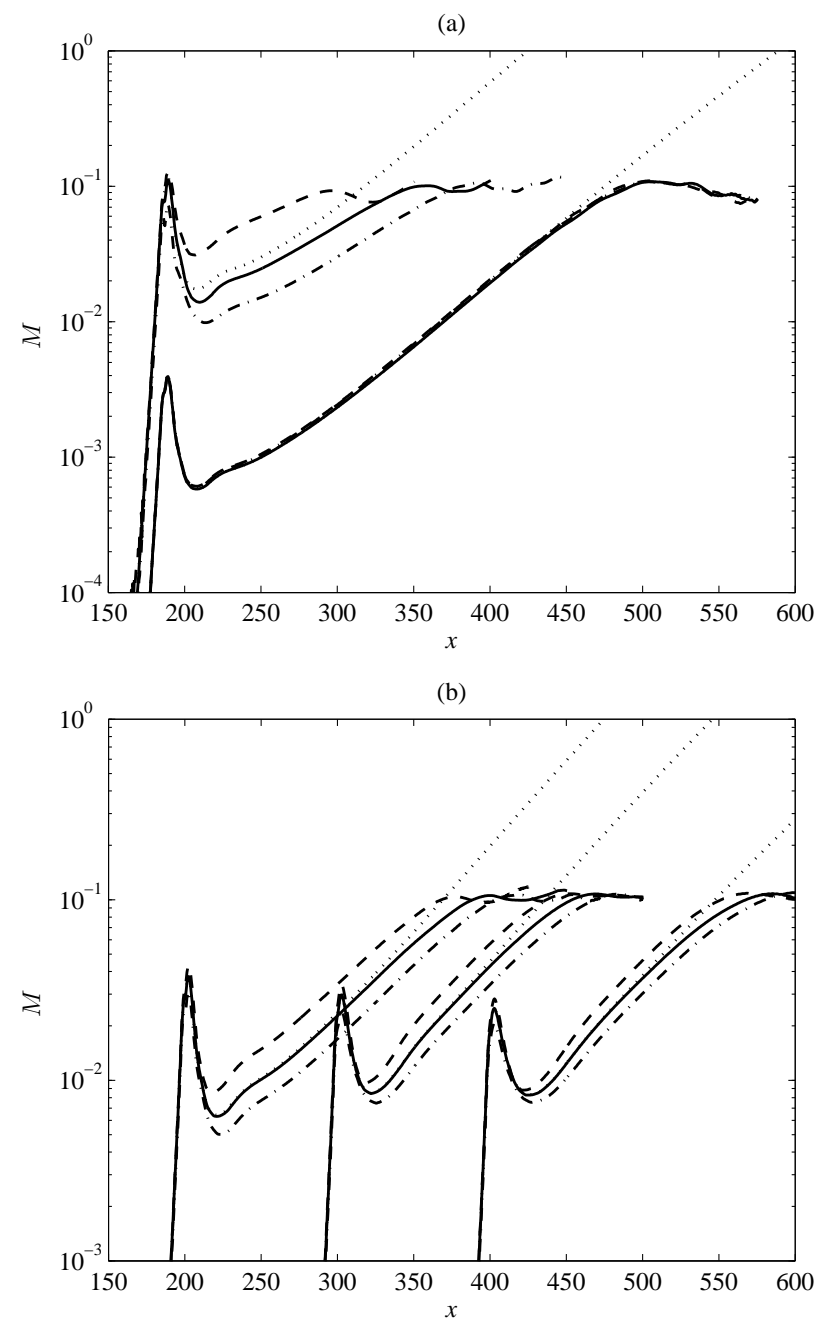

FiguRE 7. Maximum amplitudes $M$, where solid lines depict results due to forcing $\mathbf{I}$, dashed forcing $\mathbf{I I}_{\mathbf{b}}$, chain forcing $\mathbf{I I}_{\mathbf{s}}$ and dotted the linear only solution. The Reynolds number $R=500$ and the spanwise wavenumber $\beta=0.4$. (a): Centre of excitation $x_{f}=186$ and forcing amplitude $c=0.03$ (upper lines) and $c=0.001$ (lower lines). (b): Forcing amplitude $c=0.01$ and centre of excitation $x_{f}=200,300$ and 400 .

of wall excitation than the amplitude observed downstream at the stationary point. The nonlinear effects have greatly influenced both the form of the local wall forcing and the magnitude of the developing disturbance. These observations agree with the earlier analysis on the system growth and the onset of saturation for the disturbances generated with initial amplitude $c=0.03$. This would then suggest that these particular cases represent rather strong and probably physically unrealistic suction/blowing amplitude distributions.

The chord centre of the periodic forcing is varied in figure $7(\mathrm{~b})$ to illustrate the effect of the disturbance distribution on the boundary layer receptivity. Again the line types correspond to those forcing methods described above, where $c= \pm 0.01$ and $x_{f}=200$, 300 and 400. The general receptivity characteristics at each selected chord location are again similar to that described above. Method $\mathbf{I}$ has a marginal effect on the initial 

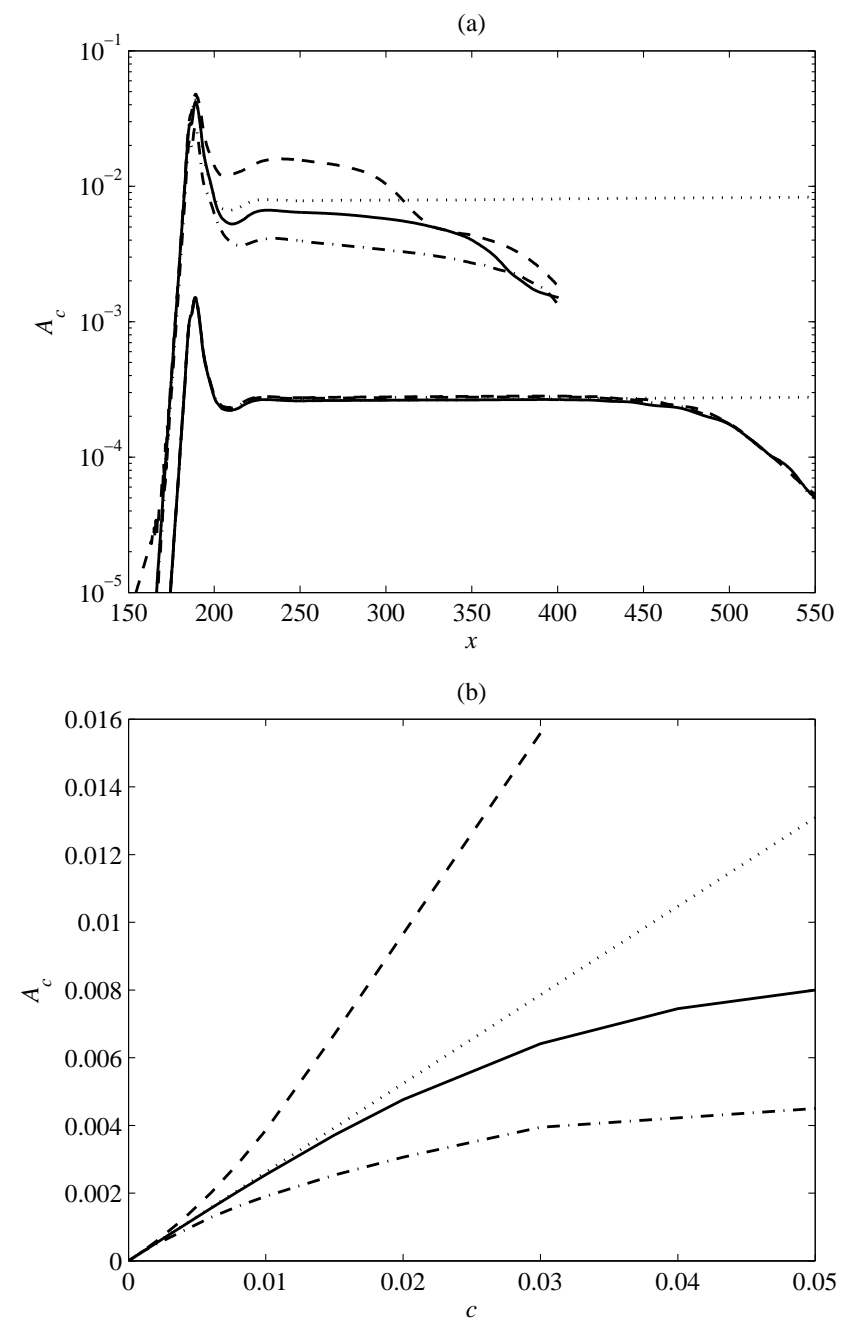

FIgURE 8. (a): Receptivity amplitudes $A_{c}$ plotted against chord length $x$. Line types correspond to that given in figure 8(a). (b): Receptivity amplitudes $A_{c}$ plotted against forcing magnitude $c \in[0.00001: 0.05]$. Again line types match that given in figure 8.

amplitude of the perturbation, whilst blowing and suction driven by forcing scheme II again significantly augments and reduces the receptivity amplitude of the disturbance. As the centre of forcing $x_{f}$ passes downstream along the chord direction, the initial amplitude of the perturbation decreases and the location of the so-called downstream stationary point increases. This behaviour may be expected as it is well known that the largest linear receptivity amplitudes are observed about neutrally stable conditions for crossflow $\left(x_{0} \sim 186\right.$ for stationary disturbances; Streett 1998; Mughal 2012). Hence in figure 7 (b), the effect of nonlinearity is greatest at $x_{f}=200$ and as $x_{f}$ is increased to larger chord positions the nonlinear effects on the receptivity amplitude diminish and the gap between the dashed (blowing) and dotted lines (suction) reduces.

\subsection{Amplitudes}

The formula for the amplitude $A_{c}$ of the crossflow instability, equation (4.4), is applied to both the linear and nonlinear perturbations in figure $7(\mathrm{a})$, which generates the plots 
shown in figure 8(a). Though the assumed PSE form of crossflow is not particularly relevant or applicable to the nonlinear stages of the evolution process, it does provide a simple means of comparing the relative sizes of the primary mode of the linear and nonlinear solutions, especially in the chordwise region immediately downstream of the forcing. The two dotted lines depict the amplitudes given for the linear only perturbations. Downstream of the periodic forcing the linear receptivity amplitudes are relatively constant over the chord length considered and their respective values are related through the size of the initial forcing $c ; A_{1}=0.26, A_{0.03}=0.0078$ and $A_{0.0001}=0.00026$. The remaining line types depict the disturbance amplitudes given by the nonlinear solutions. The perturbations generated using the smaller initial forcing are almost identical over the chord length shown and only display significant variations to the linear result for $x>450$. Downstream of this location the amplitudes decrease rapidly, which agrees with the above observations that are discussed and depicted in figure 7(a).

On the other hand, amplitudes given for the larger initial forcing are shown to vary greatly. For instance, the perturbation established using the wall blowing method II (dashed line) has a magnitude about $x=250$ that is approximately double that given by the corresponding linear disturbance, while the equivalent result for the suction only forcing (chain line) is about half the size of the linear solution. As the crossflow instability evolves and passes into the nonlinear development stages, the magnitude of the disturbance reduces and eventually all nonlinear amplitudes are smaller than the observed linear solution. This behaviour is due to the nonlinear interaction of the primary Fourier mode with its higher order harmonics. Results for the larger initial forcing are cut off at $x=400$ as this coincides with the onset of small-scale structures, which are beyond the resolution of the prescribed discretization.

Repeating the calculations given in figure 8 (a) for $x_{f}=186$ and $c$ in the range 0.00001 through to 0.05 , the four lines or curves in figure $8(\mathrm{~b})$ are generated that depict the relative sizes of $A_{c}$ against $c$ for solutions of the different forcing methods. The linear receptivity amplitudes, which are relatively constant over the chord length shown in figure $8(\mathrm{a})$, are given by the straight line $A_{c}=0.26 \mathrm{c}$. The corresponding nonlinear amplitudes are selected at the chord location $x=250$, which is chosen to give a meaningful representation of the receptivity and initial size of the disturbance. Results established by scheme II are found to be reflected about the linear receptivity line, with suction (chain) having the expected stabilising effect on receptivity amplitudes and blowing (dashed) destabilising. The third nonlinear curve (method I - solid) also shows a marked drop in the receptivity amplitude as $c$ increases, though it is not as exaggerated as that given for the suction only case.

\subsection{Stationary Point and a Semi-Log Law Relationship}

Figure 9 depicts the locations $x_{e}$ that the flow parameter $M$ attains a stationary point against both the size of the initial forcing $c$ and the initial disturbance amplitude $A_{c}$ given by the linear only analysis. A semi-log law scaling has been used along the horizontal axis in both illustrations to aid visualisation. The three nonlinear results are specified using the symbols: method I, $\square$; II, blowing, $\bigcirc$; and II, suction, $\diamond$. Open markers correspond to disturbances excited about $x_{f}=186$, while symbols with a $\times$ and + at the centre are associated with the wall forcing prescribed at $x_{f}=200,300$ and 400 , with $c=$ 0.01 and 0.001 , respectively. In figure 9 (a) the calculations associated with the wall forcing centred about $x_{f}=186$ (open markers) are found to lie along the $\left(c, x_{e}\right)$-diagonal. This is particularly true for those results computed for $c \leqslant 10^{-2}$. However, for those disturbances excited at larger values of $x_{f}$ the stationary points are shifted along the vertical axis, with the markers furthest from the diagonal representing the cases $x_{f}=400$. 

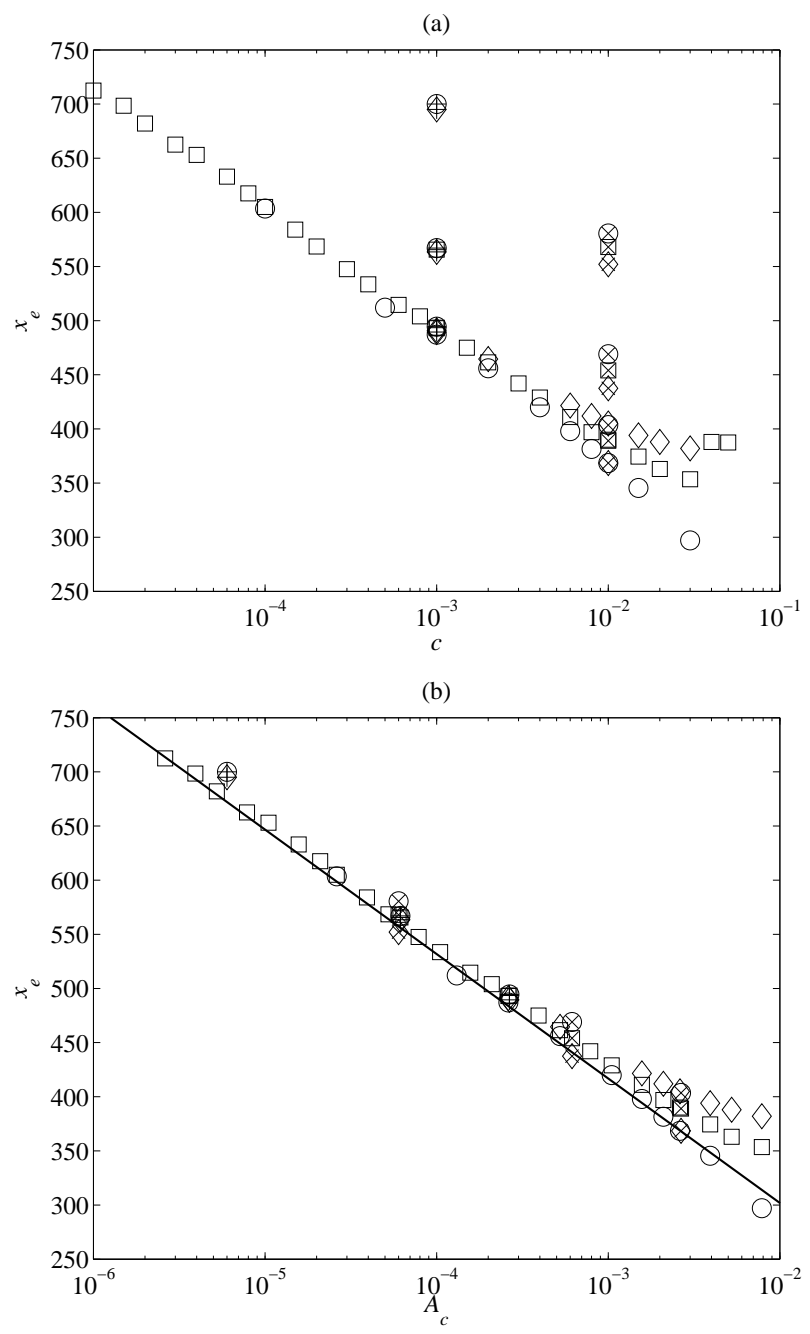

Figure 9. The chord location that the parameter $M$ attains a stationary point $x_{e}$. Plotted against (a): the amplitude of forcing $c$ and (b): the receptivity amplitude $A_{c}$ of the linear disturbance. Markers represent solutions given by the wall forcing methods I, $\square$; II, blowing, $\bigcirc$; and II, suction, $\diamond$. Open markers correspond to disturbances excited about $x_{f}=186$, while markers with $\mathrm{a} \times$ and + at the centre are associated with the wall forcing prescribed at $x_{f}=200$, 300 and 400, with $c=0.01$ and 0.001 , respectively. The solid line depicts the location that the associated linear result attains a size of 0.1 .

The relative distances in $x_{e}$ between the results of cases $x_{f}=200$ and 300 are smaller than that found between $x_{f}=300$ and 400 because receptivity is strongest about the critical conditions for crossflow instability $\left(x_{0} \sim 186\right)$; the primary Fourier mode will attain the stationary point quicker for wall forcing located near this region. As the origin $x_{f}$ of the suction/blowing distribution is shifted downstream the initial perturbation amplitude decreases and the location for the stationary point increases. The illustration then suggests that additional results for variable $x_{f}, c$ and wall distributions $h(x)$ would also be scattered throughout the $\left(c, x_{e}\right)$-plane.

All positions $x_{e}$ are plotted against the linear receptivity amplitude $A_{c}$ in figure 9(b), where markers are as before. The solid line depicts the location that the normalized 


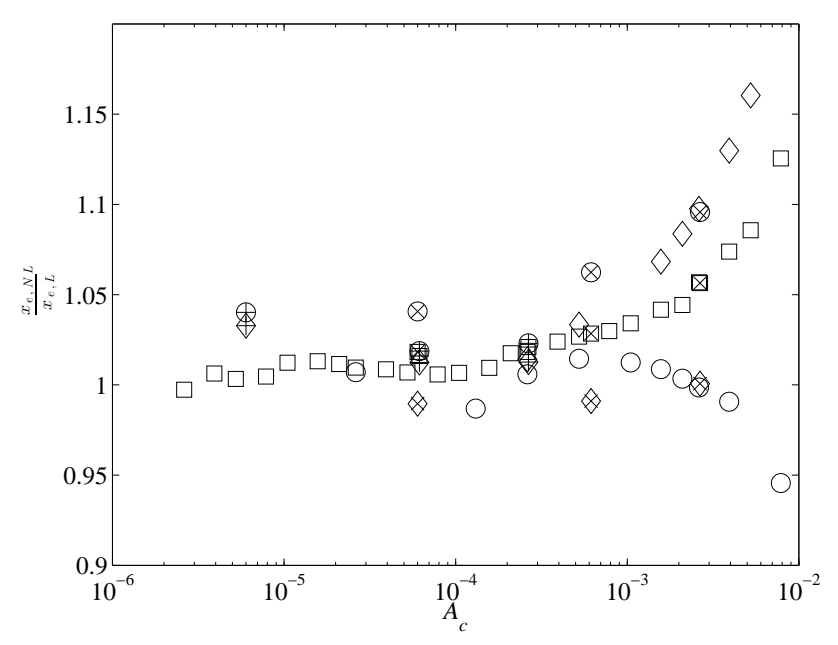

FiguRE 10. Ratio of the location that the nonlinear and linear $M$ computations attain a stationary point or amplitude 0.1. Markers are as given in Figure 9.

\begin{tabular}{|c|c|c|c|c|c|c|c|c|c|c|c|c|}
\hline$N_{y}$ & $c$ & $A_{c}$ & $E_{2} \%$ & $x_{e}$ & $c$ & $A_{c}$ & $E_{2} \%$ & $x_{e}$ & $c$ & $A_{c}$ & $E_{2} \%$ & $x_{e}$ \\
\hline Linear & 0.03 & 0.007823 & & 320 & & 0.002608 & & 370 & & 0.000261 & & 480 \\
\hline 4 & 0.03 & 0.015947 & 51.0 & 296 & 0.01 & 0.003840 & 32.1 & 378 & 0.001 & 0.000271 & 3.7 & 492 \\
\hline 6 & 0.03 & 0.017600 & 55.6 & 289 & 0.01 & 0.004177 & 37.6 & 375 & 0.001 & 0.000274 & 4.9 & 488 \\
\hline 8 & 0.03 & 0.018369 & 57.4 & 288 & 0.01 & 0.004389 & 40.6 & 373 & 0.001 & 0.000277 & 5.8 & 485 \\
\hline 10 & 0.03 & 0.019265 & 59.4 & 287 & 0.01 & 0.004558 & 42.8 & 372 & 0.001 & 0.000279 & 6.4 & 484 \\
\hline 12 & 0.03 & 0.019860 & 60.6 & 286 & 0.01 & 0.004683 & 44.3 & 372 & 0.001 & 0.000280 & 6.7 & 484 \\
\hline
\end{tabular}

TABLE 2. Results of the initial receptivity amplitude $A_{c}$, relative variation of the nonlinear amplitude with the matching linear result, $E_{2}=\frac{A_{c, N L}-A_{c, L}}{A_{c}, N L} \times 100 \%$, and stationary point position $x_{e}$, for $N_{y}=4,6, \ldots, 12$.

linear calculation attains an amplitude of 0.1 , which is consistent with the relative sizes of the nonlinear results at $x_{e}$. Interestingly all nonlinear results are located within a very small bandwidth about the solid line, including those generated using alternative forcing locations, initial amplitudes and methods. The plot suggests that for a linear receptivity amplitude $A_{c}<10^{-3}$, the chord position $x_{e}$ is located on the diagonal line given by the linear solution. As $A_{c}$ is raised to larger values, the range of $x_{e}$ increases quite significantly, with chord variations on the order of 100 for $A_{c}=10^{-2}$. However, it may still be possible to give a crude approximation for the location for $x_{e}$ using a line of best fit or the linear only calculation. A suitable fit for the linear result and nonlinear computations with $A_{c} \leqslant 10^{-3}$ is of the form

$$
A_{c} \equiv c A_{1}=\lambda e^{\gamma x_{e}},
$$

where after careful calculation the constants $\lambda$ and $\gamma$ are respectively defined as 4.2 and -0.02. For all problems considered herein the amplitude results with the initial conditions $(\omega, \beta, R)=(0,0.4,500)$ are located about this semi-log law relationship.

Figure 10 depicts the ratio of the stationary points for the nonlinear disturbances, 
$x_{e, N L}$, and the associated linear result, $x_{e, L}$. The ratio is plotted against the linear receptivity amplitude $A_{c}$ that is again drawn on a log scale axis. Markers are again as given in figure 9 . For disturbances with receptivity amplitudes $A_{c} \leqslant 10^{-3}$ the differences between the calculations of the linear and nonlinear solutions is at most $5 \%$. Thus, if the linear receptivity analysis indicates that the normalized disturbance magnitude attains a size of 0.1 about $x_{e, L}=500$, then a nonlinear simulation would give $x_{e, N L} \in[475,525]$. Hence, the simpler linear receptivity model may be used to estimate the location of disturbance processes in the more complex and time expensive nonlinear analysis. In reference to the disturbance illustrated in figure 4 above, the position of the stationary point coincides with the cross-sectional velocity illustration that is depicted in 4(c), which is marginally upstream of the location that the crossflow instability has fully rolled into a vortex structure. Similar forms are observed about $x_{e}$ for other solutions, suggesting that the stationary point appears upstream of the location described by Malik et al. (1994) and others as corresponding to full saturation.

In previous sections it was decided that $N_{y}=4$ Fourier modes was sufficient to investigate the receptivity and development of crossflow disturbances in the Swept Hiemenz boundary-layer. This decision was primarily based on the computational and time cost of simulating many disturbances based on larger values of $N_{y}$. However, as remarked upon above, disturbances to suction/blowing forcing scheme II could possible vary as $N_{y}$ is increased to larger values, causing the physical shape of forcing to asymptote towards a Dirac delta source. Hence, the results generated by forcing method II may be inaccurate, particularly for large initial forcing amplitudes $c$. Table 2 compares linear results with nonlinear calculations with varying orders of Fourier modes for the blowing only forcing (positive $c$ ). The initial receptivity amplitude of the disturbance, $A_{c}$, the relative difference with the linear solution, $E_{2}$, and the location that the primary disturbance first attains a stationary point, $x_{e}$, are documented. Following on from earlier observations, the chord location $x_{e}$ for the linear disturbance is given as the position the parameter $M$ attains a value of 0.1 . Firstly we note that the relative differences in the amplitude of the disturbance or effective receptivity are far greater than those percentage variations given for the local receptivity in table 1 . For the smallest initial forcing, $c=0.001$, the percentage differences $E_{2}$ for the $N_{y}=4$ and 12 mode cases are relatively similar with values approximately given as $E_{2}=3.7 \%$ and $6.7 \%$, respectively. Further, the variation in the location of the stationary point $x_{e}$ is very small, with linear predictions giving a very good estimate. This is also true of results given at the larger forcing, $c=0.03$, with values for $x_{e}$ again consistent for all $N_{y}$ cases considered. Although the relative percentage variation, $E_{2}$, is now much greater for all of the nonlinear simulations, the increase in receptivity amplitude from the $N_{y}=4$ to the 12 mode case is no more than $16 \%$ (based on relative differences between these two particular solutions). Thus, as a first prediction of the effects of nonlinearity on the receptivity of the crossflow development $N_{y}=4$ Fourier modes appears to give a reasonable estimate of results even at very large initial suction/blowing forcing amplitudes.

All of the results described above correspond to stationary only disturbances with the Reynolds number of the flow $R=500$. Extending the above analysis to alternative values of $R$, and a traveling crossflow disturbance, for $\omega=0.0375$ and dimensional frequency $f=20 \times 10^{-6} \mathrm{~Hz}$, we can extract similar flow properties using the methods described above. The chordwise location for the onset of the crossflow instability shifts upstream with decreasing Reynolds number and is observed to emerge for stationary disturbances about $x_{0}=137,160$ and 186 for $R=300,400$ and 500, respectively. The value of $M$ at the location that the primary disturbance attains a stationary point, $x_{e}$, is found to decrease with Reynolds number, with magnitudes typically about $0.09,0.095$ and 0.1 
(a)

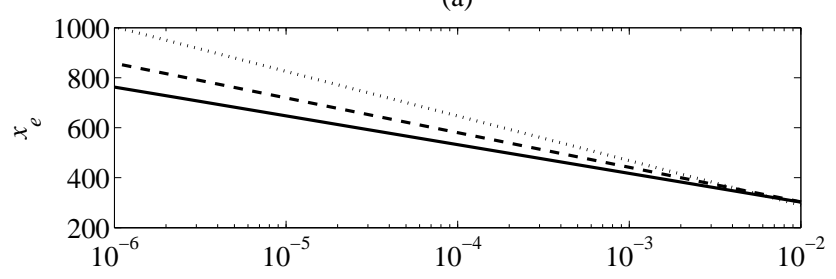

(b)

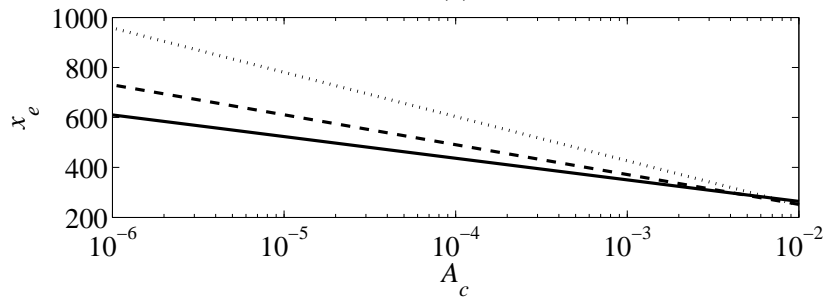

FiguRE 11. Chord location that the primary disturbance attains a stationary point against the linear receptivity amplitude $A_{c}$. Dotted lines correspond to $R=300$, dashed $R=400$ and solid $R=500$. (a): Stationary disturbances; (b): Travelling wave for $\omega=0.0375$.

for the three Reynolds numbers considered. Semi-log law plots are again constructed and depicted in figure 11 by plotting the location for $x_{e}$ against the linear receptivity amplitude $A_{c}$. Clearly the value for $x_{e}$ increases to larger values as the Reynolds number decreases, meaning that transition will generally set in further downstream. Additionally, the investigated travelling wave disturbance is more unstable than the stationary mode, as the position of the stationary point (and onset of small-scale structures) appears earlier.

\subsection{Receptivity of the Physical Perturbation}

The analysis in the preceding sections documents the flow dynamics and receptivity characteristics of the primary Fourier velocity component. Initial receptivity amplitudes and the chord location that the primary mode attains a stationary state are shown to be proportional on a linear semi-log law mapping. However, from a physical perspective it would be preferable to describe the flow properties of the real disturbance and its relationship (if any) to the receptivity amplitude. Figure 12(a) displays the disturbance response of the absolute value of three $u$-velocities as a function of $x$. Solid, dashed and chain lines correspond to the nonlinear flow solutions excited by forcing scheme $\mathbf{I}$ for the respective amplitudes $c=0.03,0.01$ and 0.001 . The dotted lines depict the corresponding linear only disturbance computations, while the three cross symbols specify the chord locations that the associated primary disturbance $u_{1}$ first attains a stationary point $x_{e}$. For these three illustrated cases the position $x_{e}$ is observed when the absolute amplitude of the physical perturbation $|u|=0.165,0.17$ and 0.23 , respectively. (Note that the results have not been normalised on the factor $R / x)$.

Figure 12(b) displays the computed values of $|u|$ at $x=x_{e}$ for all results shown in figure 9. The marker specifications are as before and a log scaling is implemented along the horizontal axis that again represents the linear receptivity amplitude $A_{c}$. Behaviour similar to that depicted in figure 9(b) is again observed; the magnitude of the nonlinear $\bar{u}$ velocity perturbations are found to lie about the solid curve that represents the size of the linear $u$-velocity at the position $x_{e}$. In particular, for $A_{c} \leqslant 10^{-3}$ results may be estimated by the curve given by the linear analysis that passes through all nonlinear results. Further, 

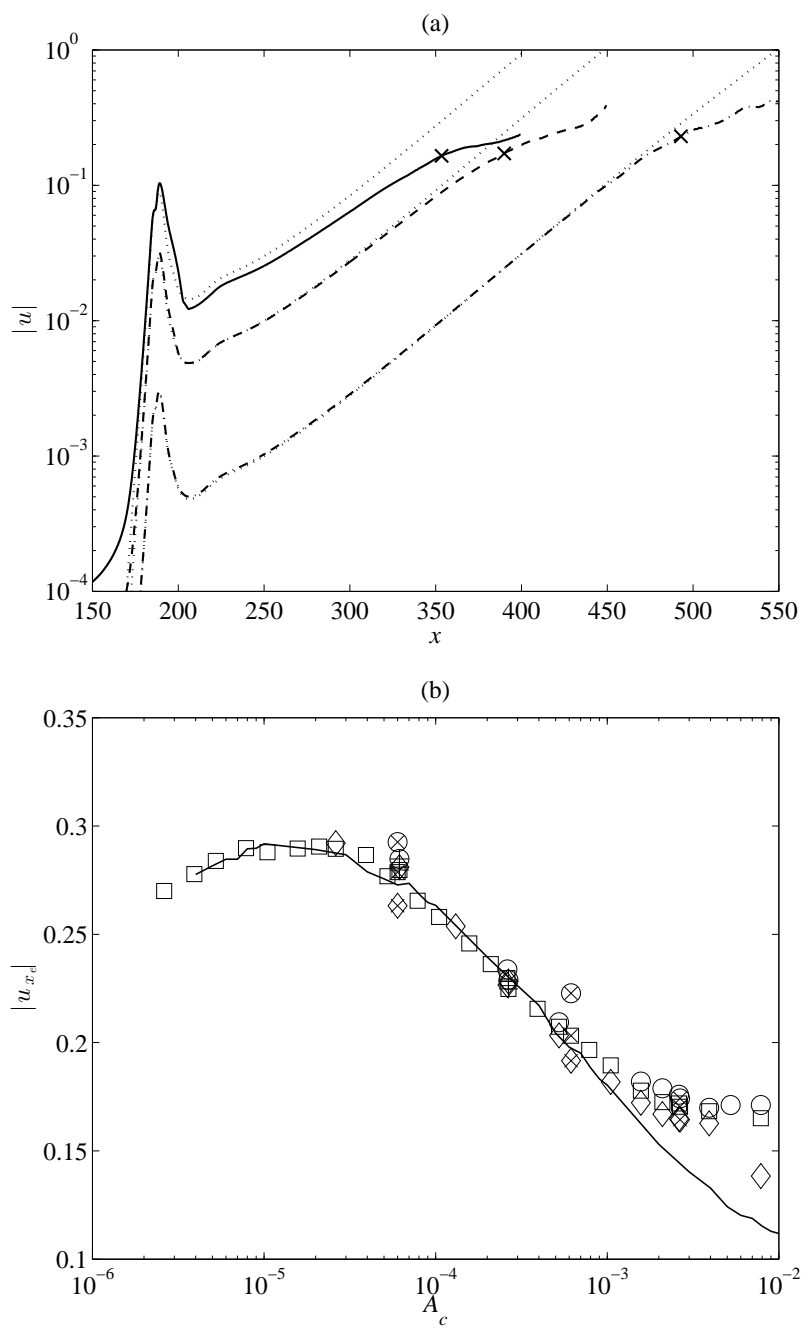

Figure 12. (a): Absolute amplitude of the $u$-velocity perturbation against the chord direction $x$. Nonlinear disturbances are excited about $x=186$ for initial forcing amplitudes $c=0.03$ (solid), 0.01 (dashed) and 0.001 (chain). (b): Magnitude of $u$-velocity component at $x_{e}$ against the linear receptivity amplitude $A_{c}$ for all results given in figure 9.

the absolute size of the disturbance $|u|$, at the chord location $x_{e}$ is observed to attain a maximum of $30 \%$ of the freestream velocity $V_{\infty}$ for a linear receptivity amplitude $A_{c} \sim 10^{-5}$.

\section{Final Remarks}

An investigation has been carried out on the effects of nonlinearity on the receptivity of crossflow in the Swept Hiemenz boundary-layer. A nonlinear vorticity formulation has been developed, extending the scheme of Davies \& Carpenter (2001) that was limited to linear disturbance development. Periodic forcing is prescribed in time and along the spanwise direction, using artificial suction/blowing strips or as bands of small holes. The effect of varying the origin and size of wall forcing are considered, as well as the effect of flow Reynolds number. Evolution of the crossflow vortex is described and shown to 
exhibit flow characteristics consistent with that depicted in earlier investigations, even though only four spanwise Fourier modes are used to generate the nonlinear results. The analysis shows that solutions generated for larger modal numbers has a relatively small impact on both the local and effective receptivity of the crossflow instability.

Suction and blowing distributed as periodic holes are shown to have a relatively large effect on the boundary-layer receptivity. Particularly those disturbances generated using large forcing magnitudes and centred near the chord location for critical crossflow instability. The absolute maximum of the primary chordwise velocity perturbation, when normalised on $R / x \equiv V_{\infty} / U_{\infty}$, is observed to attain a stationary position for an amplitude near 0.1 and is shown to be linearly proportional (on a log scaling) with the initial receptivity amplitude given by the linear analysis. This is especially true of disturbances with values $A_{c}<10^{-3}$ and all wall forcing types considered. (Extensions to wall roughness may be possible by modifying the no-slip condition and deriving similar relationships for receptivity and stationary points). This would suggest that some nonlinear flow dynamics of the crossflow vortex development may be estimated directly from the linear receptivity analysis. In terms of the size of suction/blowing wall forcing, a disturbance with initial amplitude $A_{c}=10^{-3}$ that is generated by a Gaussian shaped wall distribution about $x=186$ corresponds to an initial forcing magnitude of size $c=0.004$ or dimensionally $0.004 V_{\infty}$.

The $\log$ law relationship relating $x_{e}$ and $A_{c}$ is similar to the analysis based on the $n$-factor method for transition. Assuming that $A_{c}$ is the initial size of the disturbance, then the amplitude at $x_{e}$ is given as $0.1 x_{e} / R$, which gives the following formula for the $n$-factor:

$$
n=\ln \left(\frac{x_{e}}{10 A_{c} R}\right)
$$

However, as $x_{e}$ is located upstream of the onset of full crossflow saturation, this formula will slightly underestimate the predicted value of the $n$-factor at transition.

We thank the referees for many helpful suggestions concerning the presentation and improvement of our results. This work was carried out with the support of EPSRC through LFC-UK: Development of Underpinning Technology for Laminar Flow Control.

\section{REFERENCES}

Balachandar, S., Streett, C. L. \& Malik, M. R. 1992 Secondary instability in rotating-disk flow. J. Fluid Mech. 242, 323-347.

Bonfigli, G. \& Kloker, M. J. 2007 Secondary instabilities of crossflow vortices: validation of the stability theory by direct numerical simulation. J. Fluid Mech. 583, 229-272.

Choudhari, M. 1993 Boundary-layer receptivity due to distributed surface imperfections of a deterministic or random nature. Theoret. Comput. Fluid Dyn. 4, 101-117.

Choudhari, M. 1994 Roughness-induced generation of cross-flow vortices in three-dimensional boundary layers. Theoret. Comput. Fluid Dyn. 6, 1-30.

Choudhari, M. \& Duck, P. W. 1996 Nonlinear excitation of inviscid stationary vortex instabilities in a boundary-layer flow. In Proc. IUTAM Symp. On Nonlinear Instability and Transition in Three-Dimensional Boundary Layers, Eds. P. W. Duck and P. Hall, Kluwer Academic Publishers, pp. 409-422.

Collis, S. S. \& Lele, S. K. 1999 Receptivity to surface roughness near a swept leading edge. J. Fluid Mech. 380 (1), 141-168.

Crouch, J. D. 1993 Receptivity of three-dimensional boundary layers. In AIAA, Aerospace Sciences Meeting and Exhibit, 31 st, Reno, NV, p. 1993.

Davies, C. \& Carpenter, P. W. 2001 A novel velocity-vorticity formulation of the Navier- 
Stokes equations with applications to boundary layer disturbance evolution. J. Comp. Phys. 172, 119-165.

Federov, A. V. 1988 Excitation of waves of instability of the secondary flow in the boundary layer on a swept wing. J. Appl. Mech. Tech. Phys. 29, 643-648.

Fischer, T. M. \& Dallmann, U. 1991 Primary and secondary stability analysis of a 3D boundary-layer flow. Phys. Fluids A 3, 2378-2391.

Friederich, T. \& Kloker, M. J. 2012 Control of the secondary crossflow instability using localized suction. J. Fluid Mech. 706, 470-495.

Gregory, N., Stuart, J. T. \& Walker, W. S. 1955 On the stability of three-dimensional boundary layers with application to the flow due to a rotating disk. Phil. Trans. R. Soc. Lond. A 248, 155-199.

Hall, P., Malik, M. R. \& Poll, D. I. A. 1984 On the stability of an infinite swept attachment line boundary layer. Proc. R. Soc. Lond. A 395, 229-245.

Herbert, T. 1988 Secondary instability of boundary layers. Annu. Rev. Fluid Mech. 20, 487526.

Hosseini, S., Tempelmann, D., Hanifi, A. \& Henningson, D. S. 2013 Stabilization of a swept-wing boundary layer by distributed roughness elements. J. Fluid Mech. 718, R1.

Hunt, L. \& SARIC, W. S. 2011 Boundary-layer receptivity of three-dimensional roughness arrays on a swept-wing. In 41st AIAA Fluid Dynamics Conference and Exhibit 27 - 30 June 2011, Honolulu, Hawaii, pp. AIAA 2011-3881.

Janke, E. \& Balakumar, P. 2000 On the secondary instability of three-dimensional boundary layers. Theoret. Comput. Fluid Dyn. 14, 167-194.

KосH, W. 2000 On the spatio-temporal stability of primary and secondary crossflow vortices in a three-dimensional boundary layer. J. Fluid Mech. 456, 85-111.

Конама, Y. 1984 Study on boundary layer transition of a rotating disk. Acta Mechanica 50, 193-199.

Kohama, Y., Onodera, T. \& Egami, Y. 1996 Design and control of crossflow instability field. In Proc. IUTAM Symp. on Nonlinear Instability and Transition in Three-Dimensional Boundary Layers, Manchester, UK (ed. P. W. Duck \& P. Hall), pp. 147-156. Kluwer.

Kohama, Y., Saric, W. S. \& Hoos, J. A. 1991 A high frequency instability of crossflow vortices that leads to transition. In Proc. Roy. Aero. Soc.: Boundary-Layer Transition and Control. Cambridge.

Lovig, E., Downs, R. S. \& White, E. B. 2014 Passive laminar flow control at low turbulence levels. AIAA Journal 52, 1072-1075.

Malik, M. R. 1986 The neutral curve for stationary disturbances in rotating-disk flow. J. Fluid Mech. 164, 275-287.

Malik, M. R., Li, F. \& Chang, C. L. 1994 Crossflow disturbances in three-dimensional boundary layers: nonlinear development, wave interaction and secondary instability. $J$. Fluid Mech. 268, 1-36.

Malik, M. R., Li, F., Choudhari, M. M. \& Chang, C. L. 1999 Secondary instability of crossflow vortices and swept-wing boundary-layer transition. J. Fluid Mech. 399, 85-115.

Meyer, F. \& KLeiser, L. 1988 Numerical investigation of transition in 3D boundary layers. In AGARD Conf. Proc. 438, Fluid Dynamics of Three-Dimensional Turbulent Shear Flows and Transition, pp. 16-1.

Morkovin, M. V. 1969 On the many faces of transition. In In Viscous Drag Reduction, ed. C.S. Wells, pp. 1-31. New York: Plenum.

Mughal, S. 2012 Advanced transition prediction and development of linearised Navier-Stokes receptivity methods, validation and application. Institute for Mathematical Sciences, Imperial College London, EADS-IW ref: IW102175.

MülleR, B. \& Bippes, H. 1988 Experimental study of instability modes in a three dimensional boundary-layer. In AGARD Conf. Proc. 438, Fluid Dynamics of Three-Dimensional Turbulent Shear Flows and Transition, pp. 13-1.

NG, L. L. \& Crouch, J. D. 1999 Roughness-induced receptivity to crossflow vortices on a swept wing. Phys. Fluids 11, 432.

Obrist, D., Henniger, R. \& Kleiser, L. 2012 Subcritical spatial transition of swept Hiemenz flow. Int. J. Heat Fluid Fl. 35, 61-67. 
POLL, D. I. A. 1985 Some observations of the transition process on the windward of a long yawed cylinder. J. Fluid Mech. 150, 329-356.

Reibert, M. S., Saric, W. S., Carillo, R. B. \& Chapman, K. L. 1996 Experiments in nonlinear saturation of stationary crossflow vortices in a swept-wing boundary layer. AIAA Paper 96-0184.

SAric, W., Reed, H. \& Kerschen, E. J. 2002 Boundary-layer receptivity to freestream disturbances. Annu. Rev. Fluid Mech. 34, 291-319.

SARic, W., Reed, H. \& White, E. 2003 Stability and transition of three-dimensional boundary layers. Annu. Rev. Fluid Mech. 35, 413-440.

Schrader, L., Amin, S. \& Brandt, L. 2010 Transition to turbulence in the boundary layer over a smooth and rough swept plate exposed to free-stream turbulence. J. Fluid Mech. 646 (1), 297-325.

Schrader, L. U., Brandt, L. \& Henningson, D. S. 2009 Receptivity mechanisms in threedimensional boundary layer flows. J. Fluid Mech. 618, 209-241.

Spalart, P. R. 1988 Direct numerical study of leading edge contamination. In AGARD Conf. Proc. 438, Fluid Dynamics of Three-Dimensional Turbulent Shear Flows and Transition, pp. $5-1$.

Spalart, P. R. 1990 Direct numerical study of crossflow instability. In Proc. IUTAM Symp. On Laminar-Turbulent Transition, pp. 621-630.

Spalart, P. R. 1993 Numerical study of transition induced by suction devices. In So, R. M. C.; Speziale, C. G., Launder, B. E., (eds.): Proceedings of Conference on Near-Wall Turbulent Flows, Tempe, Arizona, U. S. A., Elsevier Science Publishers B. V.

StReett, C. 1998 Direct harmonic linear Navier-Stokes methods for efficient simulation of wave packets. AIAA Paper 1998-0784.

Tempelmann, D., Hanifi, A. \& Henningson, D. $2012 a$ Swept-wing boundary-layer receptivity. J. Fluid Mech. 700, 490-501.

Tempelmann, D., Schrader, L.-U., Hanifi, A., Brandt, L. \& Henningson, D. $2012 b$ Swept wing boundary-layer receptivity to localized surface roughness. J. Fluid Mech. 711, 516-544.

Wassermann, P. \& Kloker, M. 2002 Mechanisms and passive control of crossflow-vortexinduced transition in a three-dimensional boundary layer. J. Fluid Mech. 456 (1), 49-84.

Wassermann, P. \& KlOKer, M. J. 2003 Transition mechanisms induced by travelling crossflow vortices in a three-dimensional boundary layer. J. Fluid Mech. 483, 467-89. 